Pacific Journal of Mathematics

RADICALS OF SUPPLEMENTARY SEMILATTICE SUMS OF 


\section{RADICALS OF SUPPLEMENTARY SEMILATTICE SUMS OF ASSOCIATIVE RINGS}

\section{B. J. GARDNER}

This paper deals with the effect of radicals (in the Kurosh-Amitsur sense) on supplementary semilattice sums of rings as defined by J. Weissglass (Proc. Amer. Math. Soc., 39 (1973), 471-473). It is shown that if $\mathscr{R}$ is a strict, hereditary radical class, then $\mathscr{R}(R)=\Sigma_{\alpha \in \Omega} \mathscr{R}\left(R_{\alpha}\right)$ for every supplementary semilattice sum $R=\sum_{\alpha \in \Omega} R_{\alpha}$ with finite $\Omega$. If $\mathscr{R}$ is an $A$-radical class or the generalized nil radical class, the same conclusion holds with the finiteness restriction removed. On the other hand, if $\mathscr{R}\left(\sum_{\alpha \in \Omega} R_{\alpha}\right)=\sum_{\alpha \in \Omega} \mathscr{R}\left(R_{\alpha}\right)$ for all finite $\Omega$, then $\mathscr{R}$ is strict and satisfies

(*) $R \in \mathscr{R} \Rightarrow$ the zeroring on the additive group of $R$ belongs to $\mathscr{R}$,

a condition satisfied by both hereditary strict and $A$-radical classes.

Introduction. Semilattice sums of rings were introduced by Weissglass [11]. Let $\Omega$ be a semilattice, a commutative semigroup in which all elements are idempotent. A ring $R=\Sigma_{\alpha \in \Omega} R_{\alpha}$ is a supplemen tary semilattice sum of its subrings $R_{\alpha}$ if (i) $R^{+}=\bigoplus_{\alpha \in \Omega} R_{\alpha}^{+}$(here ( ) denotes the additive group) i.e., $R$ is a supplementary sum in the language of [3], and (ii) $R_{\alpha} R_{\beta} \subseteq R_{\alpha \beta}$ for all $\alpha, \beta \in \Omega$. Examples include direct sums, polynomial rings and semigroup rings over semilattices.

In [11], Weissglass considered the inheritance of properties by a supplementary semilattice sum $R=\sum_{\alpha \in \Omega} R_{\alpha}$ from its subrings $R_{\alpha}$. In [8], Janeski and Weissglass proved that $R$ is regular if and only if each $R_{\alpha}$ is. Their arguments need minimal modification to obtain corresponding results in which regularity is replaced by various other hereditary radical properties, including quasi-regularity, nilness and local nilpotence.

We shall be concerned with a stronger condition on a radical class $\mathscr{R}: \mathscr{R}\left(\sum_{\alpha \in \Omega} R_{\alpha}\right)=\sum_{\alpha \in \Omega} \mathscr{R}\left(R_{\alpha}\right)$ (supplementary semilattice sum) for all (finite) supplementary semilattice sums $\Sigma_{\alpha \in \Omega} R_{\alpha}$.

For general information about radical classes the reader is referred to [3]. A radical class $\mathscr{R}$ is strict if every $\mathscr{R}$-subring $S$ of a ring $R$ is contained in $\mathscr{R}(R)$, or equivalently every subring of an $\mathscr{R}$-semi-simple ring is $\mathscr{R}$-semi-simple. See [9] for further details. An A-radical class [5] is one which contains with any ring $R$ all ring $S$ with $S^{+} \cong R^{+}$. We denote the additive group of a ring by ()$^{+}$, the zeroring on an abelian group by ()$^{0} ;<$ signifies an ideal. All rings considered are associative. 


\section{The results.}

THEOREM 1. Let $\mathscr{R}$ be a radical class.

(i) If $\mathscr{R}$ is strict and hereditary, then for any supplementary semilattice sum $R=\sum_{\alpha \in \Omega} R_{\alpha}$, where $\Omega$ is finite, the rings $\mathscr{R}\left(R_{\alpha}\right)$ form a supplementary semilattice sum and $\mathscr{R}(R)=\Sigma_{\alpha \in \Omega} \mathscr{R}\left(R_{\alpha}\right)$.

(ii) If $\mathscr{R}$ is an A-radical class the same is true without the finiteness restriction on $\Omega$.

Proof. (i) We prove this by induction on $|\Omega|$, making use of the construction described in Lemmas 2 and 3 of [8]. Suppose firstly that $\Omega=\{\alpha, \beta\}, \alpha \beta=\beta$ and thus $R_{\beta}<R$. Then

$$
\mathscr{R}\left(R_{\alpha}\right) \mathscr{R}\left(R_{\beta}\right) \subseteq R \mathscr{R}\left(R_{\beta}\right) \subseteq \mathscr{R}\left(R_{\beta}\right)
$$

since $\mathscr{R}\left(R_{\beta}\right)<R \quad$ [1]. Similarly $\mathscr{R}\left(R_{\beta}\right) \mathscr{R}\left(R_{\alpha}\right) \subseteq \mathscr{R}\left(R_{\beta}\right)$ and so $\mathscr{R}\left(R_{\alpha}\right)+\mathscr{R}\left(R_{\beta}\right)$ is a supplementary semilattice sum.

We next show that $\mathscr{R}\left(R_{\alpha}\right)+\mathscr{R}\left(R_{\beta}\right)<R$. Now $R_{\alpha} \mathscr{R}\left(R_{\alpha}\right) \subseteq \mathscr{R}\left(R_{\alpha}\right)$, while $\mathscr{R}\left(R_{\alpha}\right) \subseteq \mathscr{R}(R)$ ( $\mathscr{R}$ is strict) whence $R_{\beta} \mathscr{R}\left(R_{\alpha}\right) \subset \mathscr{R}(R) \cap R_{\beta}=$ $\mathscr{R}\left(R_{\beta}\right) \quad\left(\mathscr{R}\right.$ is hereditary). Thus $R \mathscr{R}\left(R_{\alpha}\right) \subseteq \mathscr{R}\left(R_{\alpha}\right)+\mathscr{R}\left(R_{\beta}\right)$. Since $\mathscr{R}\left(R_{\beta}\right)<R$, we have $R\left[\mathscr{R}\left(R_{\alpha}\right)+\mathscr{R}\left(R_{\beta}\right)\right] \subseteq \mathscr{R}\left(R_{\alpha}\right)+\mathscr{R}\left(R_{\beta}\right)$ and a similar argument on the right completes the proof that $\mathscr{R}\left(R_{\alpha}\right)+\mathscr{R}\left(R_{\beta}\right)<R$.

Since $\mathscr{R}\left(R_{\beta}\right)<\mathscr{R}\left(R_{\alpha}\right)+\mathscr{R}\left(R_{\beta}\right)$ and

$$
\left[\mathscr{R}\left(R_{\alpha}\right)+\mathscr{R}\left(R_{\beta}\right)\right] / \mathscr{R}\left(R_{\beta}\right) \cong \mathscr{R}\left(R_{\alpha}\right) /\left[\mathscr{R}\left(R_{\alpha}\right) \cap \mathscr{R}\left(R_{\beta}\right)\right] \cong \mathscr{R}\left(R_{\alpha}\right),
$$

it follows that $\mathscr{R}\left(R_{\alpha}\right)+\mathscr{R}\left(R_{\beta}\right) \in \mathscr{R}$.

Since the sum $R_{\alpha}+R_{\beta}$ is supplementary we have isomorphisms

$$
R_{\alpha} / \mathscr{R}\left(R_{\alpha}\right) \cong\left[R / R_{\beta}\right] /\left[\left[R_{\beta}+\mathscr{R}\left(R_{\alpha}\right)\right] / R_{\beta}\right] \cong R /\left[R_{\beta}+\mathscr{R}\left(R_{\alpha}\right)\right] .
$$

It therefore follows from the exact sequence

$$
\begin{aligned}
& 0 \rightarrow\left[R_{\beta}+\mathscr{R}\left(R_{\alpha}\right)\right] /\left[\mathscr{R}\left(R_{\alpha}\right)+\mathscr{R}\left(R_{\beta}\right)\right] \rightarrow R /\left[\mathscr{R}\left(R_{\alpha}\right)+\mathscr{R}\left(R_{\beta}\right)\right] \\
& \text { II) } \\
& R_{\beta} / \mathscr{R}\left(R_{\beta}\right) \quad \rightarrow R /\left[R_{\beta}+\mathscr{R}\left(R_{\alpha}\right)\right] \rightarrow 0 \\
& \text { II) } \\
& R_{\alpha} / \mathscr{R}\left(R_{\alpha}\right)
\end{aligned}
$$

that $R /\left[\mathscr{R}\left(R_{\alpha}\right)+\mathscr{R}\left(R_{\beta}\right)\right]$ is $\mathscr{R}$-semi-simple.

Thus $\mathscr{R}\left(R_{\alpha}\right)+\mathscr{R}\left(R_{\beta}\right)=\mathscr{R}(R)$ and we have proved (i) for $|\Omega|=2$.

Now consider $|\Omega|=k$ and assume (i) for smaller semilattices. Let 
$\Omega=\Lambda \cup \theta$ where $\Lambda$ and $\theta$ are disjoint subsemilattices and $\theta$ is an ideal of $\Omega$, as in [8], Lemma 3. Then

$$
\mathscr{R}(R)=\mathscr{R}\left(R_{\Lambda}\right)+\mathscr{R}\left(R_{\theta}\right) ; \mathscr{R}\left(R_{\Lambda}\right)=\sum_{\alpha \in \Lambda} \mathscr{R}\left(R_{\alpha}\right) ; \mathscr{R}\left(R_{\theta}\right)=\sum_{\alpha \in \theta} \mathscr{R}\left(R_{\alpha}\right)
$$

and all these sums are supplementary semilattice sums. It follows that $\mathscr{R}\left(R_{\Lambda}\right)=\mathscr{R}(R) \cap R_{\Lambda}$ and

$$
\mathscr{R}\left(\mathbf{R}_{\alpha}\right)=\mathscr{R}\left(\mathbf{R}_{\Lambda}\right) \cap \mathbf{R}_{\alpha}=\mathscr{R}(\mathbf{R}) \cap \mathbf{R}_{\Lambda} \cap \mathbf{R}_{\alpha}=\mathscr{R}(\mathbf{R}) \cap \mathbf{R}_{\alpha}
$$

for every $\alpha \in \Lambda, \mathscr{R}\left(R_{\theta}\right)=\mathscr{R}(R) \cap R_{\theta}$ and $\mathscr{R}\left(R_{\alpha}\right)=\mathscr{R}(R) \cap R_{\alpha}$ for $\alpha \in \theta$. If $\alpha, \beta \in \Lambda$, then $\mathscr{R}\left(R_{\alpha}\right) \mathscr{R}\left(R_{\beta}\right) \subseteq \mathscr{R}\left(R_{\alpha \beta}\right)$ and the same conclusion is true if $\alpha, \beta \in \theta$. If $\alpha \in \Lambda$ and $\beta \in \theta$, then $\alpha \beta \in \theta$ and $\mathscr{R}\left(R_{\alpha}\right) \mathscr{R}\left(R_{\beta}\right) \subseteq R_{\alpha} R_{\beta} \subseteq R_{\alpha \beta}$. Also, $\mathscr{R}\left(R_{\alpha}\right) \subseteq \mathscr{R}(R)$, so $\mathscr{R}\left(R_{\alpha}\right) \mathscr{R}\left(R_{\beta}\right) \subseteq$ $\mathscr{R}(R)$. Thus

$$
\mathscr{R}\left(R_{\alpha}\right) \mathscr{R}\left(R_{\beta}\right) \subseteq \mathscr{R}(R) \cap R_{\alpha \beta}=\mathscr{R}\left(R_{\alpha \beta}\right) .
$$

Similarly $\mathscr{R}\left(R_{\beta}\right) \mathscr{R}\left(R_{\alpha}\right) \subseteq \mathscr{R}\left(R_{\alpha \beta}\right)$, so $\Sigma_{\alpha \in \Omega} \mathscr{R}\left(R_{\alpha}\right)$ is a supplementary semilattice sum.

Finally,

$\mathscr{R}(R)=\mathscr{R}\left(R_{\Lambda}\right)+\mathscr{R}\left(R_{\theta}\right)=\sum_{\alpha \in \Lambda} \mathscr{R}\left(R_{\alpha}\right)+\sum_{\alpha \in \theta} \mathscr{R}\left(R_{\alpha}\right)=\Sigma_{\alpha \in \Omega} \mathscr{R}\left(R_{\alpha}\right)$.

(ii) Since $R^{+}=\bigoplus_{\alpha \in \Omega} R_{\alpha}^{+}$, we have $\mathscr{R}(R)=\sum_{\alpha \in \Omega} \mathscr{R}\left(R_{\alpha}\right)$ (cf. the proof of Proposition 1.5 in [5]). The sum is a supplementary semilattice sum, because $\mathscr{R}\left(R_{\alpha}\right) \mathscr{R}\left(R_{\beta}\right) \subseteq \mathscr{R}(R) \cap R_{\alpha \beta}=\mathscr{R}\left(R_{\alpha \beta}\right)$ for any $\alpha$, $\beta \in \Omega$.

Whether or not hereditary strict radicals commute with formation of supplementary semilattice sums in general remains an open question. Some information is given by our next result, in the course of the proof of which we show that strict radical properties satisfy Weissglass' condition (F) [11].

PROPOSITION 2. Let $\mathscr{R}$ be a hereditary, strict radical class, $R=$ $\sum_{\alpha \in \Omega} R_{\alpha}$ a supplementary semilattice sum. Then $\Sigma_{\alpha \in \Omega} \mathscr{R}\left(R_{\alpha}\right)$ is a supplementary semilattice sum and an $\mathscr{R}$-ideal of $R$. Furthermore, $R / \Sigma_{\alpha \in \Omega} \mathscr{R}\left(R_{\alpha}\right) \cong \Sigma_{\alpha \in \Omega} R_{\alpha} / \mathscr{R}\left(R_{\alpha}\right)$ where the latter is a supplementary semilattice sum.

Proof. For any finite subsemilattice $\Omega^{\prime}$ of $\Omega$, we have $\mathscr{R}\left(\Sigma_{\alpha \in \Omega^{\prime}} R_{\alpha}\right)=\Sigma_{\alpha \in \Omega^{\prime}} \mathscr{R}\left(R_{\alpha}\right)$ by Theorem 1. Thus $\Sigma_{\alpha \in \Omega^{\prime}} \mathscr{R}\left(R_{\alpha}\right) \in \mathscr{R}$ for every such $\Omega^{\prime}$. But then each such $\Sigma_{\alpha \in \Omega^{\prime}} \mathscr{R}\left(R_{\alpha}\right) \subseteq \mathscr{R}\left(\Sigma_{\alpha \in \Omega} \mathscr{R}\left(R_{\alpha}\right)\right)$, so $\mathscr{R}$ contains $\Sigma_{\alpha \in \Omega} \mathscr{R}\left(R_{\alpha}\right)$ (not assumed to be a semilattice sum at this stage). 
Let $\beta, \gamma \in \Omega$ generate the semilattice $\Gamma$. Then $\Gamma$ is finite so by Theorem 1, $\mathscr{R}\left(\Sigma_{\alpha \in \Gamma} R_{\alpha}\right)=\Sigma_{\alpha \in \Gamma} \mathscr{R}\left(R_{\alpha}\right) \quad$ (supplementary semilattice sum). Hence

$$
\boldsymbol{R}_{\beta} \mathscr{R}\left(\boldsymbol{R}_{\gamma}\right) \subseteq \mathscr{R}\left(\sum_{\alpha \in \Gamma} \boldsymbol{R}_{\alpha}\right) \cap \boldsymbol{R}_{\beta \gamma}=\mathscr{R}\left(\boldsymbol{R}_{\beta \gamma}\right)
$$

and similarly $\mathscr{R}\left(R_{\gamma}\right) R_{\beta} \subseteq \mathscr{R}\left(R_{\beta \gamma}\right)$. This shows that $\Sigma_{\alpha \in \Omega} \mathscr{R}\left(R_{\alpha}\right)$ is both a supplementary semilattice sum and an ideal of $R$.

Now for any $\beta \in \Omega$, we have

$$
\left[R_{\beta}+\sum_{\alpha \in \Omega} \mathscr{R}\left(R_{\alpha}\right)\right] / \sum_{\alpha \in \Omega} \mathscr{R}\left(R_{\alpha}\right) \cong R_{\beta} /\left[R_{\beta} \cap \sum_{\alpha \in \Omega} \mathscr{R}\left(R_{\alpha}\right)\right]=R_{\beta} / \mathscr{R}\left(R_{\beta}\right)
$$

Let $I=\Sigma_{\alpha \in \Omega} \mathscr{R}\left(R_{\alpha}\right)$. It is a routine matter to show that the supplementary semilattice sum structure on $R$ induces a similar one on

$$
\sum_{\alpha \in \Omega}\left(R_{\alpha}+I\right) / I=R / I
$$

Two facts about strict hereditary radical classes may help to put the foregoing results into perspective:

(i) Such a class $\mathscr{R}$ (if nontrivial) must contain some zerorings.

Proof. If $\mathscr{R}$ doesn't contain zerorings, then every ring in $\mathscr{R}$ is idempotent. The class $\mathscr{R}^{(1)}=\{R \mid R[x] \in \mathscr{R}\}$ is also a radical class ([4], Theorem 1). Theorem 10 of [4] implies that $\mathscr{R}^{(1)}=\{0\}$, but Proposition 3.1 of [9] implies that $\mathscr{R}^{(1)}=\mathscr{R}$.

(ii) If $\mathscr{R}$ contains all zerorings, then $\mathcal{N}_{g} \subseteq \mathscr{R}$, where $\mathcal{N}_{g}$ is the generalized nil radical class of Andrunakievič and Rjabuhin [2] and Thierrin [10] (cf. Theorem 3.7 of [6]).

We can improve on Proposition 2 for $\mathscr{R}=\mathcal{N}_{g}$.

PROPOSITION 3. Let $R=\sum_{\alpha \in \Omega} R_{\alpha}$ be a supplementary semilattice sum. Then $\mathcal{N}_{g}(R)=\Sigma_{\alpha \in \Omega} \mathcal{N}_{g}\left(R_{\alpha}\right)$ (supplementary semilattice sum).

Proof. By Proposition 2, $I=\Sigma_{\alpha \in \Omega} \mathcal{N}_{g}\left(R_{\alpha}\right)$ is a supplementary semilattice sum and an $\mathcal{N}_{g}$-ideal of $R$ and $R / I$ is isomorphic to a supplementary semilatice sum $\Sigma_{\alpha \in \Omega} I_{\alpha}$ of $\mathcal{N}_{g}$-semi-simple rings. The $\mathcal{N}_{g}$-semi-simple rings are those without nonzero nilpotent elements. Suppose $x \in \Sigma_{\alpha \in \Omega} I_{\alpha}$ is a nonzero nilpotent element. Let $\Gamma$ be the (finite) subsemilattice of $\Omega$ generated by the $\alpha$ appearing in the representation of $x$. Then $x \in \Sigma_{\alpha \in \Gamma} I_{\alpha}$, so $x \in \Sigma_{\alpha \in \Gamma} \mathcal{N}_{g}\left(I_{\alpha}\right)=\mathcal{N}_{g}\left(\Sigma_{\alpha \in \Gamma} I_{\alpha}\right)$, contradicting the $\mathcal{N}_{g}$-semi-simplicity of the $I_{\alpha}$. Hence $R / I$ is $\mathcal{N}_{g}$-semisimple, so $I=\mathcal{N}_{g}(R)$. 
COROLlaRY 4. A supplementary semilattice sum $\sum_{\alpha \in \Omega} R_{\alpha}$ has no nonzero nilpotent elements if (and clearly only if) each $R_{\alpha}$ has none.

A special case of this corollary is given in [11].

Proposition 3 holds for strict, hereditary radical classes $\mathscr{R}$ such that the property of $\mathscr{R}$-semi-simplicity satisfies condition (F) of [11].

Our final result is to some extent a converse to Theorem 1.

THEOREM 5. Let $\mathscr{R}$ be a radical class such that

$$
\mathscr{R}\left(\sum_{\alpha \in \Omega} R_{\alpha}\right)=\sum_{\alpha \in \Omega} \mathscr{R}\left(R_{\alpha}\right)
$$

(supplementary semilattice sum) for every supplementary semilattice sum $\Sigma_{\alpha \in \Omega} R_{\alpha}$ with finite $\Omega$. Then

(i) $\mathscr{R}$ is strict.

(ii) $\mathscr{R}$ satisfies

$$
A \in \mathscr{R} \Rightarrow\left(A^{+}\right)^{0} \in \mathscr{R} .
$$

(iii) If in addition $\mathscr{R}$ contains all zerorings, then $\mathscr{R}$ is hereditary.

Proof. (i) Suppose $\mathscr{R}$ is not strict. Then there is a ring $Y$ with a subring $X \neq 0$ such that $X \in \mathscr{R}$ and $\mathscr{R}(Y)=0$. Define a ring $R$ by

$$
\begin{gathered}
R^{+}=Y^{+} \oplus X^{+} \\
(a, b)(c, d)=(a c+a d+b c, b d) .
\end{gathered}
$$

Then $R$ is a supplementary semilattice sum of $Y$ and $X$, while $\mathscr{R}(Y)+\mathscr{R}(X)=X$ is not an ideal of $R$ unless $X Y=0=Y X$. But this would imply that $X \triangleleft Y$, whence $\mathscr{R}(X)=0$. Not being an ideal, $\mathscr{R}(Y)+\mathscr{R}(X)$ cannot coincide with $\mathscr{R}(R)$.

(ii) If $\mathscr{R}$ does not satisfy $\left(^{*}\right)$, let $A \in \mathscr{R},\left(A^{+}\right)^{0} \notin \mathscr{R}$. Then $\mathscr{R}\left[\left(A^{+}\right)^{0}\right]^{+}$is the additive group of an ideal $I$ of $A$ (cf. Propositions 1.1 and 1.3 of [5]) and $\left(A^{+}\right)^{0} / \mathscr{R}\left[\left(A^{+}\right)^{0}\right]=\left[(A / I)^{+}\right]^{0}$ and $0 \neq A / I \in \mathscr{R}$. Thus we may assume that $\left(A^{+}\right)^{0}$ is $\mathscr{R}$-semi-simple. The ring $R$ defined by

$$
\begin{gathered}
R^{+}=A^{+} \oplus A^{+} \\
(a, b)(c, d)=(a d+b c, b d)
\end{gathered}
$$

is a supplementary semilattice sum of $\left(A^{+}\right)^{0}$ and $A$, with $\left(A^{+}\right)^{0}$ an 
ideal. Now $\mathscr{R}(A)+\mathscr{R}\left[\left(A^{+}\right)^{0}\right]=A$, while since $A^{2} \neq 0, A$ is not an ideal of $R$. Hence $A \neq \mathscr{R}(R)$.

(iii) Suppose $\mathscr{R}$ contains all zerorings, but is not hereditary. Then $\mathscr{R}$ contains a ring $Y$ with a nonzero ideal $X \notin \mathscr{R}$. Since $\mathscr{R}(X) \triangleleft Y$ [1], we have $0 \neq X / \mathscr{R}(X) \triangleleft Y / \mathscr{R}(X) \in \mathscr{R}$, so it may be assumed that $\mathscr{R}(X)=0$. Define the ring $R$ by

$$
\begin{gathered}
R^{+}=X^{+} \oplus Y^{+} \\
(a, b)(c, d)=(a c+a d+b c, b d) .
\end{gathered}
$$

This makes $R$ a supplementary semilattice sum of $X$ and $Y$, where $\mathscr{R}(X)+\mathscr{R}(Y)=Y$. But $Y$ is not an ideal of $R$ unless $X Y=0=$ $Y X$. But then $X^{2}=0$, so $X \in \mathscr{R}$.

Both $A$-radical classes (clearly) and hereditary, strict radical classes [7] satisfy $(*)$, but no example of a strict radical class satisfying $(*)$ which is neither hereditary nor an $A$-radical class is known to the author.

\section{REFERENCES}

1. T. Anderson, N. Divinsky and A. Suliński, Hereditary radicals in associative and alternative rings, Canad. J. Math., 17 (1965), 594-603.

2. V. A. Andrunakievič and Ju. M. Rjabuhin, Rings without nilpotent elements and completely simple ideals, Soviet Math. Dokl., 9 (1968), 565-568.

3. N. J. Divinsky, Rings and Radicals, Allen and Unwin, London, 1965.

4. B. J. Gardner, A note on radicals and polynomial rings, Math. Scand., 31 (1972), 83-88.

$5 . \quad$, Radicals of abelian groups and associative rings, Acta Math. Acad. Sci. Hungar., 24 (1973), 259-268.

6. - Some radical constructions for associative rings, J. Austral. Math. Soc., (to appear).

7. M. Jaegermann, Morita contexts and radicals, Bull. Acad. Polon. Sci. Sér. Sci. Math. Astronom. Phys., 20 (1972), 619-623.

8. J. Janeski and J. Weissglass, Regularity of semilattice sums of rings, Proc. Amer. Math. Soc., 39 (1973), 479-482.

9. P. N. Stewart, Strict radical classes of associative rings, Proc. Amer. Math. Soc., 39 (1973), 273-278.

10. G. Thierrin, Sur les idéaux complètement premiers d'un anneau quelconque, Acad. Roy. Belg. Bull. Cl. Sci., (5) 43 (1957), 124-132.

11. J. Weissglass, Semigroup rings and semilattice sums of rings, Proc. Amer. Math. Soc., 39 (1973), 471-478.

Received March 20, 1974.

UNIVERSITY OF TASMANIA

Hobart, Australia 


\section{PACIFIC JOURNAL OF MATHEMATICS}

\section{EDITORS}

RICHARD ARENS (Managing Editor)

University of California

Los Angeles, California 90024

\section{J. DugundJI}

Department of Mathematics University of Southern California Los Angeles, California 90007

D. Gilbarg and J. Milgram

Stanford University

Stanford, California 94305

\section{ASSOCIATE EDITORS}
E. F. BECKENBACH
B. H. NeumanN
F. WoLF
K. YoshiDA

\section{SUPPORTING INSTITUTIONS}

\author{
UNIVERSITY OF BRITISH COLUMBIA \\ CALIFORNIA INSTITUTE OF TECHNOLOGY \\ UNIVERSITY OF CALIFORNIA \\ MONTANA STATE UNIVERSITY \\ UNIVERSITY OF NEVADA \\ NEW MEXICO STATE UNIVERSITY \\ OREGON STATE UNIVERSITY \\ UNIVERSITY OF OREGON \\ OSAKA UNIVERSITY
}

\author{
UNIVERSITY OF SOUTHERN CALIFORNIA \\ STANFORD UNIVERSITY \\ UNIVERSITY OF TOKYO \\ UNIVERSITY OF UTAH \\ WASHINGTON STATE UNIVERSITY \\ UNIVERSITY OF WASHINGTON \\ AMERICAN MATHEMATICAL SOCIETY
}

The Supporting Institutions listed above contribute to the cost of publication of this Journal, but they are not owners or publishers and have no responsibility for its contents or policies.

Mathematical papers intended for publication in the Pacific Journal of Mathematics should be in typed form or offset-reproduced (not dittoed), double spaced with large margins. Underline Greek letters in red, German in green, and script in blue. The first paragraph or two must be capable of being used separately as a synopsis of the entire paper. Items of the bibliography should not be cited there unless absolutely necessary, in which case they must be identified by author and Journal, rather than by item number. Manuscripts, in duplicate, may be sent to any one of the four editors. Please classify according to the scheme of Math. Reviews, Index to Vol. 39. All other communications should be addressed to the managing editor, or Elaine Barth, University of California, Los Angeles, California, 90024.

100 reprints are provided free for each article, only if page charges have been substantially paid. Additional copies may be obtained at cost in multiples of 50 .

The Pacific Journal of Mathematics is issued monthly as of January 1966. Regular subscription rate: $\$ 72.00$ a year (6 Vols., 12 issues). Special rate: $\$ 36.00$ a year to individual members of supporting institutions.

Subscriptions, orders for back numbers, and changes of address should be sent to Pacific Journal of Mathematics, 103 Highland Boulevard, Berkeley, California, 94708.

PUBLISHED BY PACIFIC JOURNAL OF MATHEMATICS, A NON-PROFIT CORPORATION Printed at Jerusalem Academic Press, POB 2390, Jerusalem, Israel.

$$
\begin{gathered}
\text { Copyright (C) } 1975 \text { Pacific Journal of Mathematics } \\
\text { All Rights Reserved }
\end{gathered}
$$




\section{Pacific Journal of Mathematics}

\section{Vol. 58, No. $2 \quad$ April, 1975}

Zvi Artstein and John Allen Burns, Integration of compact set-valued functions . . . . . . . . . 297

Mark Benard, Characters and Schur indices of the unitary reflection group $[321]^{3} \ldots \ldots \ldots . .309$

Simeon M. Berman, A new characterization of characteristic functions of absolutely continuous

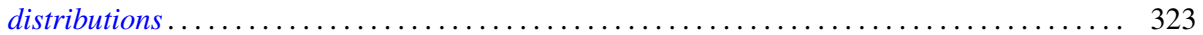

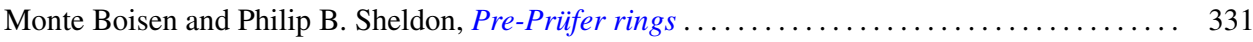

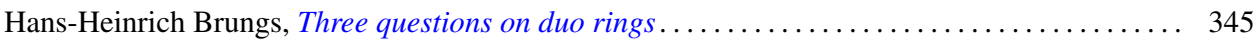

Iracema M. Bund, Birnbaum-Orlicz spaces of functions on groups................. 351

John D. Elwin and Donald R. Short, Branched immersions between 2-manifolds of higher

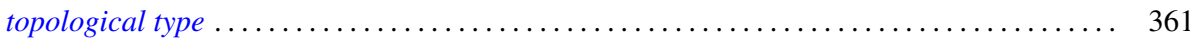

Eric Friedlander, Extension functions for rank 2, torsion free abelian groups . .......... 371

Jon Froemke and Robert Willis Quackenbush, The spectrum of an equational class of

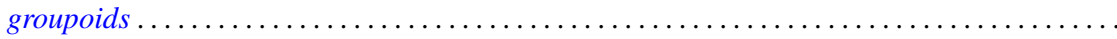

Barry J. Gardner, Radicals of supplementary semilattice sums of associative rings ...........

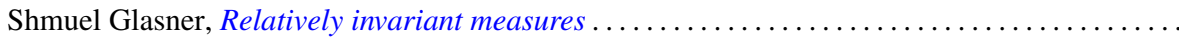

George Rudolph Gordh, Jr. and Sibe Mardesic, Characterizing local connectedness in inverse

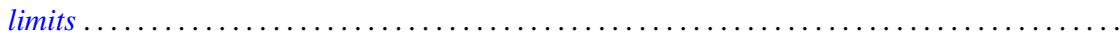

Siegfried Graf, On the existence of strong liftings in second countable topological spaces......

Stanley P. Gudder and D. Strawther, Orthogonally additive and orthogonally increasing

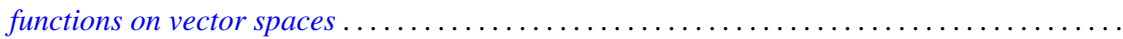

Darald Joe Hartfiel and Carlton James Maxson, A characterization of the maximal monoids and

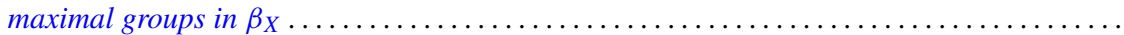

Robert E. Hartwig and S. Brent Morris, The universal flip matrix and the generalized faro-shuffle. .

William Emery Haver, Mappings between ANRs that are fine homotopy equivalences. .

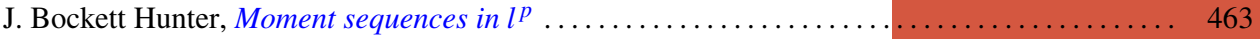

Barbara Jeffcott and William Thomas Spears, Semimodularity in the completion of a poset.... 467

Jerry Alan Johnson, A note on Banach spaces of Lipschitz functions . . . . . . . . . . . . 475

David W. Jonah and Bertram Manuel Schreiber, Transitive affine transformations on

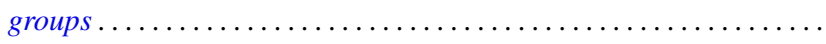

Karsten Juul, Some three-point subset properties connected with Menger's characterization of

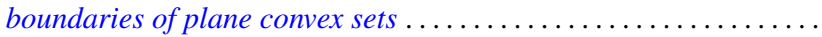

Ronald Brian Kirk, The Haar integral via non-standard analysis . . . . . . . . . . . . . 517

Justin Thomas Lloyd and William Smiley, On the group of permutations with countable

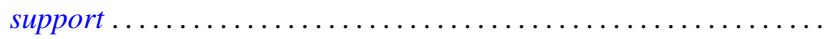

Erwin Lutwak, Dual mixed volumes .................................. 531

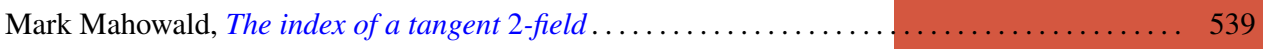

Keith Miller, Logarithmic convexity results for holomorphic semigroups . . . . . . . . . . . . 549

Paul Milnes, Extension of continuous functions on topological semigroups . . . . . . . . . . 553

Kenneth Clayton Pietz, Cauchy transforms and characteristic functions ................ 563

James Ted Rogers Jr., Whitney continua in the hyperspace $C(X) \ldots \ldots \ldots \ldots \ldots \ldots \ldots \ldots .569$

Jean-Marie G. Rolin, The inverse of a continuous additive functional . . . . . . . . . . . . 585

William Henry Ruckle, Absolutely divergent series and isomorphism of subspaces . ........ 605

Rolf Schneider, A measure of convexity for compact sets . ..................... 617

Alan Henry Schoenfeld, Continous measure-preserving maps onto Peano spaces .......... 627

V. Merriline Smith, Strongly superficial elements .......................... 643

Roger P. Ware, A note on quadratic forms over Pythagorean fields . . . . . . . . . . . . . . 651

Roger Allen Wiegand and Sylvia Wiegand, Finitely generated modules over Bezout rings . . . . 655

Martin Ziegler, A counterexample in the theory of definable automorphisms . . . . . . . . . 665 